\title{
Modelling the Relaxation Characteristics of Current- and Optically Controlled Superconducting Switching Elements
}

\author{
Jan Waśkiewicz and Jerzy Gołębiowski
}

\begin{abstract}
The work presents results of the research on the relations between temperature and resistance relaxation times of thin films of the $\mathrm{YBa}_{2} \mathrm{Cu}_{3} \mathrm{O}_{7-x}$ superconductor deposited on substrates with different thermal conductivity $\left(\mathrm{SrTiO}_{3}, \mathrm{MgO}\right.$, $\mathrm{Al}_{2} \mathrm{O}_{3}$ ) and the duration of optical and current pulses (ranging from $5 \mathrm{~ns}$ to $1 \mathrm{~ms}$ ) and the temperature level of the superconductor film. The research was conducted by means of the numerical modelling method of the resistance response in the superconducting element. The research findings can be used for developing thin-film structures of switching elements in order to optimize the process of selecting materials and the film thickness adjusted to working modes of these elements.
\end{abstract}

Keywords-high- $\mathrm{T}_{c}$ superconductors, optical switches, currentoptical pulses, relaxation characteristics.

\section{INTRODUCTION}

$\mathbf{T}$ HE phenomena of rapid transition of thin films of high$\mathrm{T}_{c}$ superconductors (HTS) from the superconducting state into the normal metal state and backwards (the $\mathrm{S} \leftrightarrow \mathrm{N}$ transition) induced by current and optical radiation allow to construct various opto- and cryoelectronic components operating at the liquid nitrogen temperature: high-sensitivity bolometers, switching elements, fault current limiters, microwave filters, etc [1], [2]. While thin films are transmitted to the normal metal state $(\mathrm{N})$, a great influence on the time of superconductivity reproduction is exerted by processes of heat generation and propagation in thin-film structures, which has been noted in many experimental studies [3]-[5]. Nevertheless, determining the relation between the response of HTS films and thermal processes based on the data obtained in experimental research is very difficult, as during experiments several mechanisms changing the film resistance occur, and also because HTS films have diverse parameters in different studies depending on the type of substrate, which has a significant impact on the response characteristics. In works [6]-[9] in order to examine the influence of thermal processes on relaxation characteristics of HTS thin-film elements, numerical modelling methods of the thermal mechanism of the film response on current and optical interaction were applied, which enabled to examine the impact of heat generation on the response characteristics depending on particular factors.

This paper was prepared at Białystok Technical University within a framework of the S/WE/3/08 project sponsored by the Ministry of Science and Higher Education, Poland.

J. Waśkiewicz and J. Gołebiowski are with the Faculty of Electrical Engineering, Bialystok Technical University, Wiejska 45d, 15-351 Bialystok, Poland (e-mail: j.waskiewicz@pb.edu.pl; goleb@we.pb.edu.pl).

\section{Physical-Mathematical Model}

The computational models [10], [11] were constructed in conformity with the geometrical and temporal conditions typical of the switching components under consideration. At present, switching processes are usually studied using elements based on $\mathrm{YBa}_{2} \mathrm{Cu}_{3} \mathrm{O}_{7-x}$ films. High speed can be achieved only with high quality films with a low temperature width of the superconducting transition $\left(\Delta T_{c}=0.5-1 \mathrm{~K}\right)$. These films must be single crystalline with a crystallographic $c$ axis perpendicular to the plane of the film. In order to improve the quality of films on some substrates, such as $\mathrm{MgO}$, a buffer layer (sublayer) of material that is crystallographically matched to the structure of the HTS film is first deposited. An HTS film is produced in the form of a microbridge with the following characteristics: thickness $4=0.1-0.2 \mu \mathrm{m}$, width $w=10-100 \mu \mathrm{m}$, and length $l=0.1-15 \mathrm{~mm}$. The substrates usually have a thickness of $0.2-0.5 \mathrm{~mm}$ and the buffer thickness is $0.1-0.5 \mu \mathrm{m}$.

An equilibrium state develops in several stages in an HTS film. For example, under optical irradiation, the absorbed photons initially transfer energy to electrons at the penetration depth of the radiation, $\sim 100 \mathrm{~nm}$ [12]. Electrons with energies on the order of $1 \mathrm{eV}$ thermalize primarily through electronelectron collisions over times $\sim 300$ fs [13] and penetrate to an estimated [14], [15] depth of 500-800 $\mathrm{nm}$ at liquid nitrogen temperatures. As the excess energy of the electrons falls below $0.1 \mathrm{eV}$, thermalization through electron-phonon collisions begins to predominate [16] with a time constant of $\sim 1$ ps [17], [18]. The phonon component of the thermal conductivity along the $c$ axis in $\mathrm{YBa}_{2} \mathrm{Cu}_{3} \mathrm{O}_{7-x}$ single crystals [19] is roughly two orders of magnitude greater than the electron component, i.e., heat is mainly carried by phonons whose mean free path at $90 \mathrm{~K}$ is on the order of $1 \mathrm{~nm}$. These characteristic relaxation times suggest that a local thermodynamic state develops in $\mathrm{YBa}_{2} \mathrm{Cu}_{3} \mathrm{O}_{7-x}$ films over times shorter than 10 ps.

Other mechanisms (besides bolometric) for conductivity switching in these control regimes $(\tau>0.1 \mathrm{~ns} ; d \geq 100$ $\mathrm{nm}$ ) either do not act at all, or end earlier (their effect can be characterized by the appearance of an effective resistance), or make a small contribution to the resulting response. Resistance owing to heating of the electron subsystem (Testradi effect) can have an independent effect only in films with thicknesses $d<30 \mathrm{~nm}$ [17], while in thicker films for times $\tau>10$ ps, the effect reduces to heating of the film. An electrical response caused by the kinetic inductance of a microbridge 
has been analyzed in a number of papers [20], [21] and it has been shown that it is also caused by rapid heating of the film, so it can be referred to as an inductive-bolometric effect. Estimates [22] show that the amplitude of this response is at least an order of magnitude smaller than the purely bolometric response.

Current disruption of the superconductivity of narrow films by currents much above critical also takes place over $\sim 10 \mathrm{ps}$ (on the order of the electromagnetic diffusion time) [23] and for modeling of the response to current pulses lasting $>0.1 \mathrm{~ns}$, can be taken into account by introducing a resistance in the leading edge of the response pulse.

Given these factors, the basis of our mathematical model [10] for the response of HTS films to light and current pulses was assumed to be a thermal response mechanism, and the temperature distributions were calculated using the nonstationary heat conduction equations.

Besides the superconductor 1, sublayer 3, and substrate 5 layers, the geometric model of an HTS element shown in Fig. 1 includes layers 2 and 4 that model the region of thermal contacts between the HTS film and the buffer layer, as well as between the buffer layer and the substrate. It is necessary to introduce contact layers because experimental data indicate that there is a large thermal resistance $\mathrm{Rb}$ in the contact regions. For example, the interface between an epitaxial $\mathrm{YBa}_{2} \mathrm{Cu}_{3} \mathrm{O}_{7-x}$ film and an $\mathrm{MgO}$ (or $\mathrm{SrTiO}_{3}$ ) substrate has been found [24] to have a thermal resistance $\mathrm{R}_{b} \approx 10^{-8} \mathrm{~m}^{2} \cdot \mathrm{K} / \mathrm{W}$ comparable to the thermal resistance of an HTS film of thickness 100-200 nm. The film 1 is acted on by optical radiation with the energy density $\mathrm{E}_{F}$ and a transport current $I$ flows along the film.

For the characteristic geometric dimensions of microbridges, when the length and width of the HTS film are at least 50 times its thickness, in the calculations of pulsed heating of the film a one-dimensional model can be used with the assumption that heat propagates along the $x$ axis perpendicular to the plane of the HTS film. The system of time dependent heat conduction equations for the layers of a film structure have the following general form:

$$
c_{k} \rho_{k} \frac{\partial T}{\partial t}=\lambda_{k} \frac{\partial^{2} T}{\partial x^{2}}+p_{k}(x, t, T)
$$

where $k$ is the number of the layer of the film structure (Fig. $1)$; $T$ is the temperature; $t$ is the time; $x$ is the coordinate; $c_{k}, \rho_{k}$, and $\lambda_{k}$ are the specific heat, density, and thermal conductivity, respectively, of the material in the $k$-th layer; and, $p$ is the volume power density of heat released in the plane at position $x$.

Since dielectric, optically transparent substrates and buffer layers are usually used in film structures, we may assume that heat is released only in the superconducting layer and that (1) will be homogeneous $(p=0)$ for layers 2-5.

The thermophysical parameters $c_{k}, \rho_{k}$, and $\lambda_{k}$ were assumed to be constant, since in the temperature range from $77 \mathrm{~K}$ (liquid nitrogen) to $90 \mathrm{~K}$ (the onset of the superconducting transition) of greatest interest for analyzing the response characteristics, the errors associated with neglecting

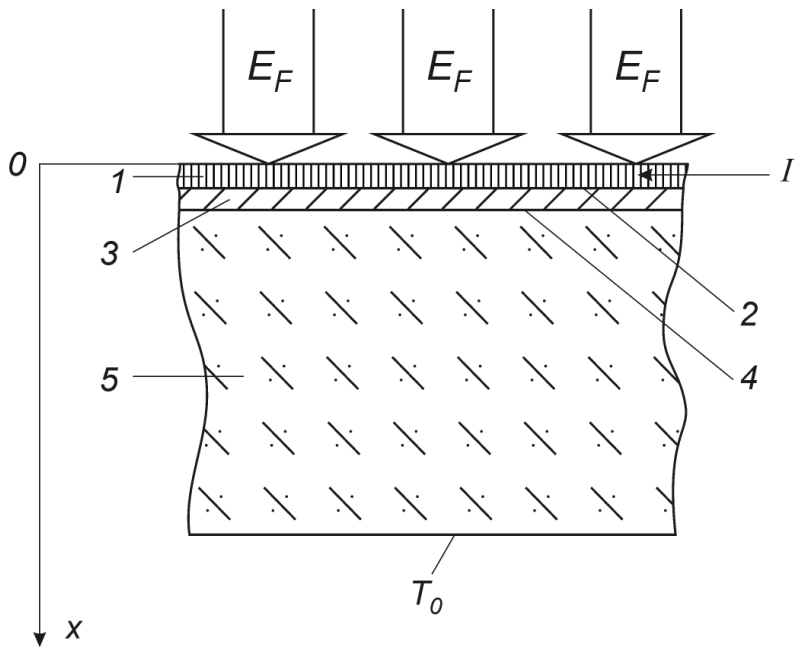

Fig. 1. Physical-geometrical model of an HTS film element: 1 - HTS film, 3 - buffer layer, 5 - substrate, 2 and 4 - thermal contact regions; $E_{F}$ is the energy density of the radiation, $I$ is the current, and $T_{0}$ is the thermostat temperature.

the temperature dependences of these parameters is less than $15 \%$.

The volume power of the heat source in the HTS layer, $p$, is made up, in general, of the power $p_{l}$ generated by the absorption of the light and by Joule heating, $p_{j}$, and depends on the time, position, and temperature of the superconductor, i.e.,

$$
p(x, t, T)=p_{l}(x, t)+p_{j}(t, T) .
$$

Since the penetration depth of visible and near infrared light in $\mathrm{YBa}_{2} \mathrm{Cu}_{3} \mathrm{O}_{7-x}, \sim 100 \mathrm{~nm}$ [12], is commensurate with the thickness of the HTS film, on taking the transmission of the film into account, the radiative power $q$ absorbed per unit area of the film is given by

$$
q(t)=q_{0}(t)\left(1-R_{v}\right)\left(1-e^{-\kappa d_{1}}\right),
$$

where $R_{v}$ is the spectral reflectivity of the film, $\kappa$ is the absorption coefficient of the film material, and $q_{0}(t)$ is the flux density of the incident radiation.

Since the diffusion mean free path for fast electrons exceeds the HTS layer thickness, we may assume that on a time scale $t>100 \mathrm{ps}$, the heat sources are uniformly distributed over the film thickness, i.e.,

$$
p_{l}(x, t)=\underset{x}{\operatorname{const}(t)}=\frac{q(t)}{d_{1}} .
$$

The time variation of the laser pulses was approximated by a rectangular or gaussian shape. The shape of pico- and nanosecond light pulses is usually assumed to the rectangular, since in this case the response is determined mainly by the total energy delivered to the film. The amplitude of the pulse was then determined from the density of the total energy of the optical signal, $q_{0}=E_{F} / \tau i$.

The volume Joule heating power $p_{j}$ is determined assuming a uniform distribution of the current over the film cross section, which is valid after the disruption of the $S$ state, i.e.,

$$
p_{j}(t, T)=j^{2}(t) \cdot \rho(T, j),
$$


where $j$ is the current density and $\rho$ is the specific electrical resistivity of the HTS film.

Modeling the current interaction is a complex task, because in the region of the phase transition $\rho$ depends on the current density, as well as on the temperature. The following algorithm was carried out in the model. The onset temperature of the $N \rightarrow S$ transition, $T_{N}$, and $\rho\left(T_{N}\right)$ are assumed constant. The $j_{c}(T)$ curve, which is linear over a wide temperature range near the superconducting transition, is essentially used to solve the inverse problem of determining the transition temperature $T_{c}$ from the current density in the film. The $\rho(T)$ curve between these points was approximated by different functions selected by analyzing the experimental pulsed current-voltage characteristics of the HTS films. The function $\rho(T, j)$ used in this paper, which contains the square of the sine [11], is plotted in Fig. 2.

Therefore, the effect of the transport current was taken into account in the model, on one hand, through its effect on $T_{c}$ and $\rho$ at a given temperature and, on the other, through the heat release (5). In the front of the current pulse a transition from one $\rho(T)$ curve to the other at a fixed temperature is carried out in the program (Fig. 2); this is fully justified, since the time to disrupt the superconducting state with a current is $\sim 10 \mathrm{ps}$, an order of magnitude shorter than the shortest pulsed interactions considered here. The resulting value of $\rho$ is used to calculate the resistance of the film assuming a uniform distribution of resistivity. This modelling approach is suitable for analyzing the responses to current pulses well above critical, sufficient to bring all or most of the length of the microbridge into a normally conducting state in the leading edge of the current pulse.

In the complete heat conduction boundary value problem, equation (1) for the five layers is supplemented by boundary and initial conditions. At low temperatures, heat removal from the outer surface of the film into the surrounding medium is negligible and for $x=0$ we can assume Neumann (type II) boundary conditions,

$$
\left.\frac{\partial T(t)}{\partial x}\right|_{x=0^{+}}=0
$$

Type IV boundary conditions hold for the inner interfaces of

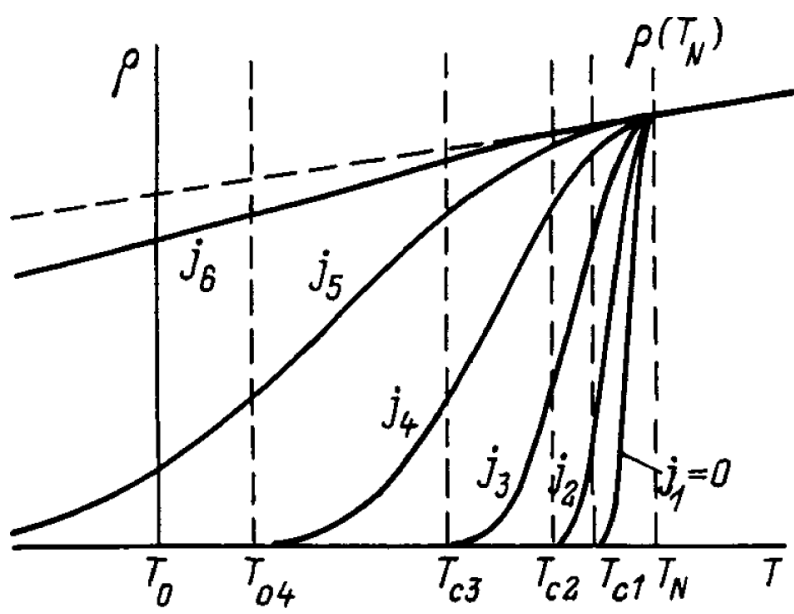

Fig. 2. Approximating the $\rho(T, j)$ curve by piecewise continuous functions. the layers, $x_{k}$; these represent equality of the fluxes on the left and right,

$$
\left.\lambda_{k} \frac{\partial T(t)}{\partial x}\right|_{x_{k}^{-}}=\left.\lambda_{k+1} \frac{\partial T(t)}{\partial x}\right|_{x_{k}^{+}},
$$

where $k=1 \ldots 4$ is the number of a layer from the HTS film to the layer adjacent to the substrate, while

$$
x_{k}=\sum_{m=1}^{k} d_{m}
$$

represents the coordinate of the interface of the layers, where $d_{m}$ is the thickness of the layers.

For the substrate surface $x_{5}$, which adjoins the heat sink, a Dirichlet (type I) condition is specified: $T\left(x_{5}, t\right)=T_{0}$. The initial condition $T(x, 0)=T_{0}$ reflects the fact that at time $t=0$ the entire structure is at the temperature of the thermostat.

The heat conduction boundary value problem formulated here is a system of one-dimensional, inhomogeneous, and nonstationary differential equations that are linear in the thermophysical properties of the materials and boundary conditions, but have a highly nonlinear volume heat source $p_{j}$ in the first layer. The problem can only be solved numerically, with the entire set of parameters of the structure, material, and external interactions. The method chosen was a finite difference method employing a purely implicit Euler scheme. The system of algebraic equations was linearized by Newton's method. This yields a system of equations with a tridiagonal matrix which is rapidly solved by factorization method.

In the program for executing the model, the parameters of the electrical circuit for hookup of the HTS film are also established.

\section{RELAXATION CHARACTERISTICS AND THEIR ANALYSIS}

Below there are calculation and analysis results of the relations between temperature $\left(\tau_{T}\right)$ and resistance $\left(\tau_{R}\right)$ relaxation times of thin $\mathrm{YBa}_{2} \mathrm{Cu}_{3} \mathrm{O}_{7-x}$ films on various $0.5 \mathrm{~mm}$-thick substrates and the duration of rectangular optical and current pulses $\tau_{i}$, as well as between the HTS film temperature level. While calculating temperature fields, the thermal resistance of

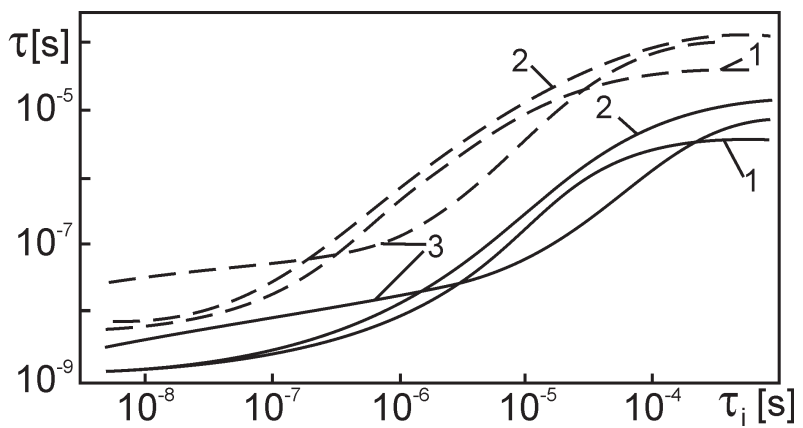

Fig. 3. Relations between the temperature relaxation time $\tau_{T}$ (the broken line) and the resistance relaxation time $\tau_{R}$ (the solid line) and the duration of optical pulses $\tau_{i}$ for $\mathrm{YBa}_{2} \mathrm{Cu}_{3} \mathrm{O}_{7-x}$ films deposited on the following substrates: $\mathrm{Al}_{2} \mathrm{O}_{3}$ (curves 1), $\mathrm{MgO}$ (2) and $\mathrm{MgO}$ with a buffer layer $\mathrm{SrTiO}_{3}(3)$. 
contact areas between the structure layers, assumed to amount to $10^{-8} \mathrm{~m}^{2} 2 \mathrm{~K} / \mathrm{W}$, was taken into account, and the cryostat temperature was assumed to be $T_{o}=78 \mathrm{~K}$.

Fig. 3 shows calculation results of temperature and resistance relaxation times, $\tau_{T}$ (the broken line) and $\tau_{R}$ (the solid line), depending on the duration of optical pulses $\left(\tau_{i}\right)$. The $\tau_{T}$ and $\tau_{R}$ relaxation times are determined by: the time of the excess temperature drop to the level of $1 / e$ of its maximum value obtained at the moment of the pulse termination and the time of the resistance drop in the HTS film to $10 \%$ of the maximum value respectively. The presented results are applicable to HTS films deposited on the following substrates: $\mathrm{Al}_{2} \mathrm{O}_{3}$ (the structure marked Y/Al, curves 1), $\mathrm{MgO}$ (Y/Mg, curves 2) and $\mathrm{MgO}$ with the $0.5 \mu$ m-thick $\mathrm{SrTiO}_{3}$ buffer layer (Y/Sr/Mg, curves 3). Geometric, electrical and optical parameters of the HTS film in all structures were identical: thickness $d_{1}=0.2 \mu \mathrm{m}$; width $w=20 \mu \mathrm{m}$; length $l=2 \mathrm{~mm}$; critical temperature at the end of superconducting transition for low direct currents $T_{c}=87 \mathrm{~K}$; temperature width of the $\mathrm{S} \leftrightarrow \mathrm{N}$ transition $\Delta T_{c}=3 \mathrm{~K}$; specific resistance: for $T_{N}=T_{c}+\Delta T_{c}=90 \mathrm{~K} \rho_{N}=0.1 \mathrm{~m} \Omega \cdot \mathrm{cm}$ and for $T=150 \mathrm{~K}$ $\rho(150)=0.15 \mathrm{~m} \Omega \cdot \mathrm{cm}$. The thermophysical parameters of HTS structure materials employed in the calculations are listed in Tab. I. The energy EF of optical pulses was selected in such a way so that at the moment of termination of a pulse with any length, the temperature in the mid-plane of the HTS film, being the basis for calculating the resistance response, increased to the same maximum value of $T_{m}=90 \mathrm{~K}$. While applying such a method of heating the superconducting transition range will fall on the steepest initial part of the temperature drop curve in the HTS film, and the $\tau_{T}$ and $\tau_{R}$ times will have lower values than in case of high values of the $T_{m}$ temperature.

Weak relations between the $\tau_{T}$ and $\tau_{R}$ relaxation times and $\tau_{i}$ (Fig.3) within the short-pulse range can be explained by the fact that the conditions of heat abstraction from the HTS film to the substrate become slightly changed as a result of a shallow depth of heat penetration into the substrate (at the order of one micrometer) at the $\tau_{i}$ time. Moreover, the changes in $\tau_{T}$ and $\tau_{R}$ are correlated with the changes in thermal diffusivity of materials on which HTS films are deposited: the higher thermal diffusivity is, the faster relaxation. Along with the increase in the depth of heat penetration into the substrates as $\tau_{i}$ gets higher, relaxation times, $\tau_{T}$ and $\tau_{R}$ become longer. Differences in the course of the curves for the $\mathrm{Y} / \mathrm{Mg}$ and $\mathrm{Y} / \mathrm{Sr} / \mathrm{Mg}$ structures should be also taken into account: starting from certain values of $\tau_{i}$, the $\tau_{T}$ and $\tau_{R}$ times will be shorter for the structure with the $\mathrm{SrTiO}_{3}$ buffer layer, which is a poor thermal conductor, than for the structure without a buffer layer. It is related to the fact that while heating HTS films to the same temperature, $T_{m}$, because of the temperature drop in the buffer layer in the $\mathrm{Y} / \mathrm{Sr} / \mathrm{Mg}$ structure with a lower light pulse energy, the substrate will be heated to lower temperatures than in the $\mathrm{Y} / \mathrm{Mg}$ structure. The subsequent temperature drop in the HTS film to the $\left(T_{m}-T_{o}\right)$ /e level, when HTS and near-surface films of the substrate become isothermal, is faster in a structure with a buffer layer. As $\tau_{i}$ approximates the time of obtaining the stationary state $(0.15 \mathrm{~ms}$ for the $\mathrm{MgO}$ substrate $)$ the rise in relaxation times becomes slower, and with further increase of
TABLE I

The THERMOPHySiCAl PARAMETERS OF HTS STRUCTURES EMPLOYED IN THE CALCULATIONS

\begin{tabular}{cccc}
\hline \hline Material & $\begin{array}{c}\lambda_{k} \\
\mathrm{~W} / \mathrm{m} \cdot \mathrm{K}\end{array}$ & $\begin{array}{c}c_{k} \\
\mathrm{~J} / \mathrm{kg} \cdot \mathrm{K}\end{array}$ & $\begin{array}{c}\rho_{k} \\
\mathrm{~kg} / \mathrm{m}^{3}\end{array}$ \\
\hline $\mathrm{YBa}_{2} \mathrm{Cu}_{3} \mathrm{O}_{7-x}$ & 5 & 150 & 6350 \\
$\mathrm{SrTiO}_{3}$ & 5 & 200 & 5000 \\
$\mathrm{MgO}$ & 340 & 150 & 3600 \\
$\mathrm{Al}_{2} \mathrm{O}_{3}$ & 900 & 100 & 4000 \\
\hline \hline
\end{tabular}

$\tau_{i}$, the relaxation times remain steady.

The curves presented in Fig. 3 also show that maintaining the $T_{m}=T_{N}$ condition in accordance with the non-linearity of the $\rho(T)$ relation, the resistance relaxation time is shorter than the temperature relaxation time by the order of magnitude. Additionally, for the HTS film deposited on substrates with good thermal conductivity $\left(\mathrm{Al}_{2} \mathrm{O}_{3}, \mathrm{MgO}\right)$, for pulses shorter than $10^{-7} \mathrm{~s}$, the resistance relaxation time is much shorter than the time-constant of the heat diffusion through that layer (at the order of $10^{-8} \mathrm{~s}$ for the thickness of $0.2 \mu \mathrm{m}$ ). Analogical relations were also obtained for current pulses.

In any thin-film structure heating the HTS film above the $T_{N}$ temperature leads to a rapid growth of the time of the film return to the superconducting state $(\mathrm{S})$ with practically unchanged temperature relaxation time of the film in a given structure. Relaxation delay in the HTS film takes place because in the "overheating" mode $\left(T>T_{N}\right)$ the rapid drop in its temperature after termination of heating falls on the gentle segment of the change in the film resistance in the normal metal state, and the range of superconducting transition, $\Delta T_{c}$ falls on the gentle segment of the temperature drop. It is clearly visible in Fig. 4, depicting temporal characteristics for the film temperature (a) and resistance (b) while being affected by $1 \mu \mathrm{s}$ current pulses with amplitudes (in $\mathrm{mA}$ ) given next to particular curves. It should be noted that while heating the HTS film above the $T_{N}$ temperature, $\tau_{R}$ times may considerably exceed specific times of the film temperature drop. This phenomenon is particularly conspicuous in elements where low-quality films are applied (with low $T_{c}$ and $j_{c}$ values). The courses presented

(a)

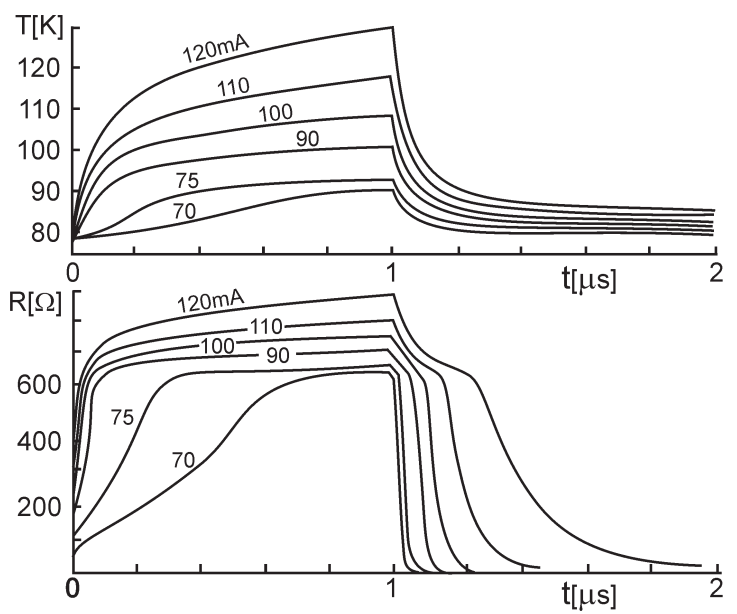

Fig. 4. Temporal characteristics for temperature (a) and resistance (b) of the HTS film while being affected by $1 \mu$ s current pulses with different amplitudes. 
(a)

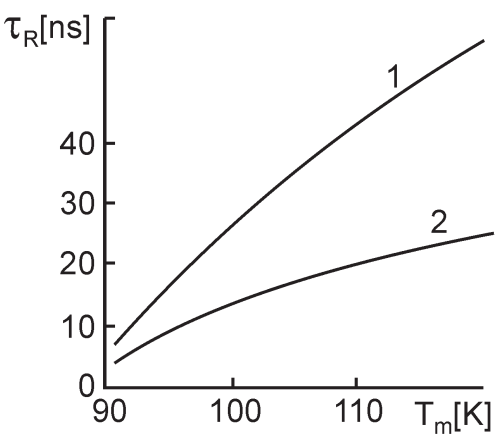

(b)

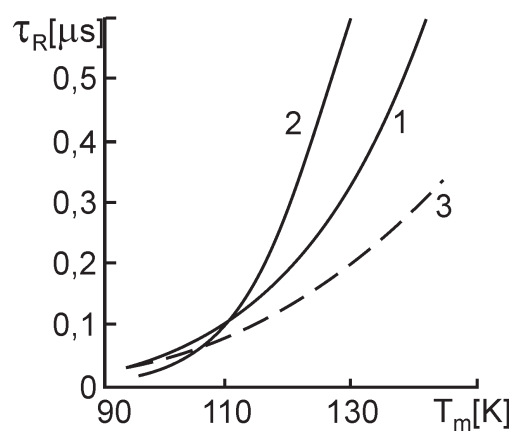

Fig. 5. Relation between the resistance relaxation time $\left(\tau_{R}\right)$, and the temperature $\left(T_{m}\right)$ of the HTS film in the following structures: Y/Sr/Mg (1), Y/Al (2) and Y/Sr/Al (3) for current pulses of 8ns (a) and $1 \mu \mathrm{s}$ (b).

in Fig. 4, were determined for the HTS film deposited on the $\mathrm{MgO}$ substrate with the $0.5 \mu \mathrm{m}$-thick $\mathrm{SrTiO}_{3}$ buffer layer with the parameters corresponding to the data presented in [23]: $d_{1}=0.2 \mu \mathrm{m} ; w=30 \mu \mathrm{m} ; l=2 \mathrm{~mm} ; \mathrm{T} c=84 \mathrm{~K} ; T_{N}=$ $90 \mathrm{~K} ; \rho_{N}=0.2 \mathrm{~m} \Omega \cdot \mathrm{cm} ; j_{c}(78)=5 \cdot 10^{5} \mathrm{~A} / \mathrm{cm}_{2}$. Despite the fact that in the experimental research [23] the HTS film was incorporated into an electric circuit with a voltage source, the calculation results correspond to experiment results sufficiently well.

The calculations demonstrated that relations between the duration of the superconducting state restoration and the temperature, $T_{m}$, of the HTS film, as well as the dependencies from the pulse duration described above, are equivocally determined by thermal diffusivity of the substrates. Fig. 5 shows the $\tau_{R}\left(T_{m}\right)$ relations for the following HTS film $\left(d_{1}=0.2 \mu \mathrm{m}\right.$; $\left.T_{c}=87 \mathrm{~K} ; T_{N}=90 \mathrm{~K} ; j_{c}(78)=10^{6} \mathrm{~A} / \mathrm{cm}^{2}\right)$ deposited on the $\mathrm{MgO}$ substrate with the $\mathrm{SrTiO}_{3}$ buffer layer (curves 1) and on a material with higher thermal conductivity, $\mathrm{Al}_{2} \mathrm{O}_{3}$, without

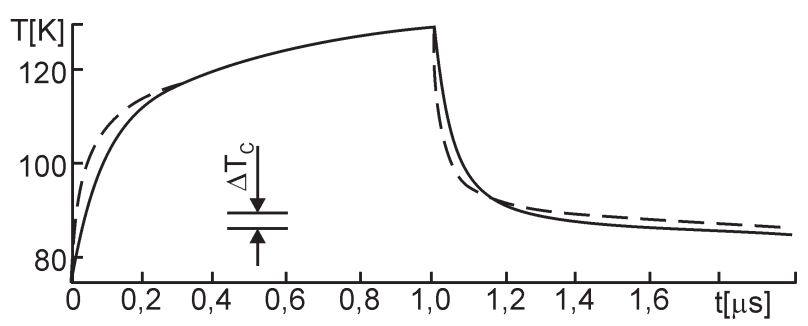

Fig. 6. Temperature courses of the HTS film deposited on the Al2O3 substrate (the broken line) and the $\mathrm{MgO}$ substrate with the $\mathrm{SrTiO} 3$ buffer layer (the solid line).

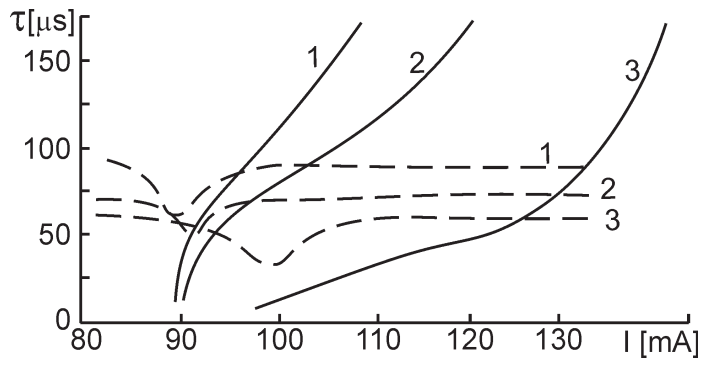

Fig. 7. Relations between the temperature relaxation time $\tau_{T}$ (the broken line) and the resistance relaxation time $\tau_{R}$ (the solid line), and the current pulse amplitude $I$ for the following structures: $\mathrm{Y} / \mathrm{Sr} / \mathrm{Mg}$ (curves 1), Y/Sr/Al (2) and Y/Al (3).

a buffer layer (curves 2) and with the $\mathrm{SrTiO}_{3}$ buffer layer (curve 3), calculated for current pulses lasting $8 \mathrm{~ns}$ (a) and $1 \mu \mathrm{s}$ (b). For short pulses (Fig.5a) and a relatively low level of the film heating, the high thermal conductivity of $\mathrm{Al}_{2} \mathrm{O}_{3}$ makes the resistance relaxation of the film very quick. Whereas for long pulses (Fig.5b), along with the rise in the $T_{m}$ temperature, the resistance relaxation time of the film deposited on the $\mathrm{Al}_{2} \mathrm{O}_{3}$ substrate becomes longer than in the $\mathrm{Y} / \mathrm{Sr} / \mathrm{Al}$ structure, and even in $\mathrm{Y} / \mathrm{Sr} / \mathrm{Mg}$, which can be explained by a different course of the film cooling in various structures.

The $\mathrm{T}(\mathrm{t})$ curves presented in Fig. 6 show a situation, where the film cooling on the $\mathrm{Al}_{2} \mathrm{O}_{3}$ substrate (the broken line) within the superconducting transition range is lower than in the $\mathrm{Y} / \mathrm{Sr} / \mathrm{Mg}$ structure (the solid line). The courses of these curves indicate that for lower values of $T_{m}$ the $\Delta T_{c}$ range of the $\mathrm{S} \leftrightarrow \mathrm{N}$ transition will fall on the initial segments of the temperature drop, on which the film cooling rate on the $\mathrm{Al}_{2} \mathrm{O}_{3}$ substrate will be greater than in the $\mathrm{Y} / \mathrm{Sr} / \mathrm{Mg}$ structure, and the resistance relaxation time will be relatively lower.

Apart from the effects of thermal states of thin-film structures on relaxation characteristics of HTS films discussed above, we should note the lowering of the temperature relaxation time of the films while being current controlled as far as the values of the current amplitudes are concerned, corresponding to the development of thermal instability in the process of the film heating (the curve for the $70 \mathrm{~mA}$ current is shown in Fig. 4). This phenomenon is the more conspicuous the greater thermal resistance of the substrate and the pulse duration are, which can be explained by shortening of the film effective heating time in relation to duration of the current pulse [6]. Examples of the $\tau_{T}(I)$ relation with a hollow caused by the effect of thermal instability are shown in Fig. 7 (the broken line) for the following structures: $\mathrm{Y} / \mathrm{Sr} / \mathrm{Mg}$ (curve 1), Y/Sr/Al (curve 2) and Y/Al (curve 3). In Fig. 7 there are also $\tau_{R}(I)$ characteristics (solid lines). The calculations were done for $\tau_{i}=1 \mu \mathrm{s}$ with the same parameters of the HTS film for which the relations in Figures 5 and 6 were obtained. The shift of the characteristics to the right as thermal resistance of the structures becomes lower is related to the reduction in the heating temperature of the films, $T_{m}$, at the same current amplitudes. 


\section{CONCLUSIONS}

The proposed thermal model of the resistance response of thin-film HTS elements on optical and current pulses, which was validated in experimental research within the pulse duration range from 100ps to $10 \mathrm{~ms}$ [10], [20], [23], can be applied for developing thin-film structures of HTS elements in order to optimize the process of selecting materials and the film thickness adjusted to working modes of these elements. It can be also used for analysing experiment results in order to detect the appearance of other mechanisms changing the resistance of HTS films.

\section{REFERENCES}

[1] N. Sekiya, H. Harada, Y. Nakagawa, S. Ono, and S. Ohshima, "Design and Fabrication of Bandwidth Tunable HTS Transmit Filter Using $\pi$ shaped Waveguides," Physica C, vol. 470, no. 20, pp. 1499-502, 2010.

[2] J. Liu, R. R. Mansour, and M. M. A. Salama, "A New Low-loading HTS Current Switch Triggered by RF Signals," IEEE Transactions on Applied Superconductivity, vol. 15, no. 2, pp. 1994-1997, 2005.

[3] T. Koyama, K. Kaiho, I. Yamaguchi, and S. Yanabu, "Study of Superconductor Recovery Time Characteristics and High-speed Reclosing of Electromagnetic Repulsion Sitch," Electrical Engineering in Japan, vol. 175, no. 3, pp. 12-19, 2011

[4] J. Wosik, L. M. Xie, and R. Grabovickic, "Thermal Effects in Microwave Current-induced Weak Link Switching in YBCO Thin Films," Superconductor Science and Technology, vol. 22, no. 10, p. 105003(7), 2009.

[5] L. Parlato, G. P. Pepe, D. Pan, C. D. Lisio, V. Pagiarulo, and A. Cosentino et al., "Time-resolved Optical Characterization of Proximized Nanobilayers for Ultrafast Photodetector Applications," Journal of Physics. Conference Series, vol. 97, p. 012317(6), 2008.

[6] J. Waśkiewicz, "Optimization of the Optical Pulse Energy for Switches Based on Thin Films of High- $\mathrm{T}_{c}$ Superconductors," Electronics and Telecommunications Quarterly, vol. 54, no. 2, pp. 127-133, 2008.

[7] _ " "Optimal Control Characteristics of Superconductor Switching Elements Driven by Current and Laser Pulses," Proceedings of SPIE, vol. 6599, pp. 05.1-5, 2007.

[8] _ " "Control Mode Optimization for Superconductor Switches Driven by Current-optical Pulses," Proceedings of SPIE, vol. 5948, pp. 21.1-7, 2005.

[9] — "Reasons of Switch-off Time Decrease in Superconductor Switches With a Buffer Layer," Physica C, vol. 372-376, no. 2, pp. 630-633, 2002.

[10] A. V. Mezenov and Y. Vaskevich, "Model of Current and Optical Conductivity Switching in $\mathrm{YBa}_{2} \mathrm{Cu}_{3} \mathrm{O}_{7-x}$ Films," Technical Physics, vol. 44, no. 10, pp. 1198-202, 1999.
[11] A. V. Mezenov, A. V. Yankevich, and J. Waśkiewicz, "Modeling the Electrical Response of Thin Films of High-Temperature Superconductors to Laser and Current Pulses," Journal of Optical Technology, vol. 66, no. 8, pp. 749-754, 1999 .

[12] H. Yasuoka, H. Mazaki, and T. Terashima et al., "Optical Absorption Spectra of Single-crystal $\mathrm{YBa}_{2} \mathrm{Cu}_{3} \mathrm{O}_{y}$ Film," Physica $C$, vol. 175, no. 1-2, pp. 192-196, 1991

[13] S. G. Han, Z. V. Vardeny, and O. Symko et al., "Femtosecond Dynamics of Quasi-Particles in $\mathrm{YBa}_{2} \mathrm{Cu}_{3} \mathrm{O}_{7-\delta}$ Superconductor Films," IEEE Transactions on Magnetics, vol. 27, no. 2, pp. 1548-51, 1991, pt. 2.

[14] E. M. Gershenzon, G. N. Gol'tsman, and A. Sergeev et al., "Picosecond Response of YBaCuo Films to Electromagnetic Radiation," in Proc. Eur. Conf. High- $T_{c}$ thin films and single crystals, Ustroń, 1989, pp. 457-462.

[15] P. H. Ballentine, A. M. Kadin, and W. R. Donaldson et al., "Optically Triggered Switching of Superconducting $\mathrm{YBa}_{2} \mathrm{Cu}_{3} \mathrm{O}_{7-\delta}$ Thin Films," Proceedings of SPIE, vol. 1287, pp. 134-143, 1990.

[16] N. Bluzer, "Temporal Relaxation Measurements of Potoinduced Nonequilibrium in Superconductors," Journal of Applied Physics, vol. 71, no. 3, pp. 1336-48, 1992.

[17] M. Gershenzon, V. Golovlev, and I. Kedich et al., "Direct measurement of electron-phonon coupling characteristics in $\mathrm{YBa}(\mathrm{sub} 2)$ $\mathrm{Cu}(\mathrm{sub} 3) \mathrm{O}$ (sub7) by femtosecond laser spectroscopy," JETP Letters, vol. 52, no. 11 , pp. 602-605, 1990.

[18] J. M. Chwalek, C. Uher, and J. F. Whitaker et al., "Femtosecond Optical Absorption Studies of Nonequilibrium Electronic Processes in High- $\mathrm{T}_{c}$ Superconductors," Applied Physics Letters, vol. 57, no. 16, pp. 1696-98, 1990.

[19] S. J. Hagen, Z. Z. Wang, and N. Ong, "Anisotropy of the Thermal Conductivity of $\mathrm{YBa}_{2} \mathrm{Cu}_{3} \mathrm{O}_{7-y}$," Physical Review B, vol. 40, no. 13, pp. 9389-92, 1989.

[20] F. A. Hegmann and J. S. Preston, "Origin of the Fast Photoresponse of Epitaxial $\mathrm{YBa}_{2} \mathrm{Cu}_{3} \mathrm{O}_{7-\delta}$ Thin Films," Physical Review B, vol. 48, no. 21, pp. 16 023-39, 1993.

[21] D. Ladret, B. Cabon, and T. Dinh et al., "Measurements and Modeling of the Kinetic Iductance in High Critical Temperature Superconducting Transmission Lines," IEEE Transactions on Applied Superconductivity, vol. 5, no. 3, pp. 3482-87, 1995 .

[22] B. S. Karasik, M. A. Zorin, and I. I. Milostnaya et al., "Subnanosecond Switching of $\mathrm{YBaCuO}$ Films Between Superconducting and Normal States Induced by Current Pulse," Journal of Applied Physics, vol. 77, no. 8, pp. 4064-70, 1995.

[23] A. B. Kozyrev, T. B. Samoilova, and S. Y. Shaferova, "Superconductivity: physics, chemistry, technique," 1993.

[24] C. D. Marshall, A. Tokmakoff, and I. M. Fishman et al., "Thermal Boundary Resistance and Diffusivity Measurements on Thin $\mathrm{YBa}_{2} \mathrm{Cu}_{3} \mathrm{O}_{7-x}$ Films with $\mathrm{MgO}$ and $\mathrm{SrTio}_{3}$ Substrates Uing the Transient Grating Method," Journal of Applied Physics, vol. 73, no. 2, pp. 850-7, 1993. 